\title{
QTL Analysis of Agronomic Traits in Rice Using SSR and AFLP Markers
}

\author{
Dina KEBRIYAEE, Mojtaba KORDROSTAMI*, Mohammad \\ Hossein REZADOOST, Habibollah Samizadeh LAHIJI \\ University of Guilan, Faculty of Agricultural Sciences, Rasht, Iran; kordrostami009@gmail.com (corresponding author)
}

\begin{abstract}
A quantitative trait is a measurable trait that depends on the cumulative action of many genes and their interaction with the environment that can vary among individuals over a given range to produce a continuous distribution of phenotypes. The identification and mapping of QTLs for such traits has great significance for rice breeders. In this study, a genetic analysis of quantitative trait loci (QTLs) affecting 11 agronomical traits was performed using an $\mathrm{F}_{2}$ population of a cross between two Oryza sativa ssp. indica type cultivars, 'Domsiah' and 'IR44', comprising 160 plants. A total of 22 QTLs were detected for 11 traits, including two for plant height (PH), one for full grain per panicle (FG), two for flag leaf width (FLW), three for 1000-grain weight (TGW), two for number of panicles per plant (NP), three for flag leaf length (FL), two for peduncle length (Pd), one for panicle exertion (PE), two for grain yield (GY), two for basal panicle length (BPL) and two for awn length (AL).
\end{abstract}

Keywords: AFLP, agronomical traits, quantitative trait loci, SSR

\section{Introduction}

The rapid progress in the development of polymorphic molecular markers has led to the intensive use of QTL mapping in genetic study for quantitative traits (Wang $e t$ al., 2007).

In general, QTLs are identified by correlating genetic variation with trait variation; a significant correlation between genotype and phenotype suggests that DNA status helps determine trait expression (Grisel, 2000). The two general goals of QTL mapping in plants are to (a) increase the biological knowledge of the inheritance and genetic architecture of quantitative traits, both within a species and across related species, and (b) identify markers that can be used as indirect selection tools in breeding (Semagn, 2010).

Numerous genetic studies of quantitative traits have been done in a large number of plant species. With the advent of DNA molecular markers, QTL mapping has become a routine strategy for the discovery of genes involved in complex quantitative traits. Thousands of QTL have been mapped for important agronomical traits in rice (Xing et al., 2008). Several researches have been made about the yield related QTLs. Wang et al. (2010) conducted a quantitative trait loci (QTL) analysis of 150 rice recombinant inbred lines (RILs) derived from a cross between two cultivars, Oryza sativa ssp. indica cv. '93-11' and Oryza sativa ssp. japonica cv. 'Nipponbare' and detected 49 QTL with phenotypic effect ranging from 3.2 to $46.0 \%$ for 14 agronomics traits. Five QTL of relatively large effect (14.6-46.0\%) were located on small genomic regions, where strong candidate genes were found. Gomez et al. (2006) mapped QTLs linked to physio-morphologi$\mathrm{cal}$ and plant production traits under drought stress in the field by evaluating $177 \mathrm{~F}_{6}$ recombinant inbred (RI) lines of 'Bala' $\times$ 'Azucena' under rainfed conditions in the target environment (TE). A total of 24 QTLs were identified for various traits under stress, which individually explained 4.6 to $22.3 \%$ phenotypic variation. Bei-Bei et al. (2003) mapped quantitative trait loci (QTLs) for harvest index, biomass, grain yield, sink capacity and plant height using a $\mathrm{DH}$ population containing $81 \mathrm{DH}$ lines from an indicajaponica cross of rice. A total of 21 significant main effect QTLs and 9 pairs of epistatic loci were detected. Of these, three detected QTLs for grain yield collectively accounted for $42 \%$ of the phenotypic variation with a LOD of 7110 .

\section{Materials and methods}

\section{Plant material and field evaluation}

The plant material consisted of $160 \mathrm{~F}_{2}$ individuals derived from a cross between two Oryza sativa ssp. indica type cultivars, 'Domsiah' ('DOM') and 'IR44' that differs in all agronomic traits. 'Domsiah' is an Iranian native variety with a long grain. It has a black spot on one end, which gives it the name. It is cultivated mainly in the Guilan province of Iran. 'Domsiah' is especially valued for its aroma (Singh et al., 1996). This Aromatic variety is a high yielding variety too. 'IR44' is an improved rice from IRRI, dwarf and drought and cold resistant. The experiment was carried out in a randomized complete block design, with a spacing of $25 \times 25 \mathrm{~cm}$ at the Rice Research Institute of Iran (Rasht). Observations on $\mathrm{F}_{2}$ plants were taken at the time of flowering and harvesting and the mean values were used 
for the QTL analysis. The total of 12 yield related traits were evaluated including full grain per panicle (FG), flag leaf width (FLW), 1000-grain weight (TGW), number of panicles per plant (NP), flag leaf length (FL), peduncle length $(\mathrm{Pd})$, plant height $(\mathrm{PH})$, panicle exertion $(\mathrm{PE})$, total weight of panicle (TWP), grain yield (GY), basal panicle length (BPL) and awn length (AL).

\section{SSR and AFLP analysis}

Fresh leaves were used for DNA extraction according to CTAB method (Rogers et al., 1988) with slightly modifications. In this study a set of 105 SSR markers were tested to find the polymorphic markers between the parents (McCouch et al., 2002). The sequences of each of the primer pairs were downloaded from http://www.gramene.org/ microsat/ssr.html. Among these, 51 polymorphic markers were chosen. These markers were distributed over all twelve rice chromosomes. PCR reaction was carried out in $10 \mu \mathrm{l}$ reaction mixture containing $50 \mathrm{ng}$ of template (Genomic DNA), $20 \mathrm{mM} \mathrm{MgCl}, 4 \mathrm{mM}$ of dNTPs, $1 \mathrm{U}$ of Taq and $2 \mu \mathrm{M}$ of each primer in $1 \mathrm{x}$ reaction buffer. The amplification reaction was performed in the Eppendorf Master cycler with an initial denaturation for 4 minute at $94^{\circ} \mathrm{C}$, then 35 cycles: 40 seconds denaturation at $94^{\circ} \mathrm{C} ; 40 \mathrm{sec}-$ onds annealing at $52^{\circ} \mathrm{C} ; 2$ minute extension at $72^{\circ} \mathrm{C}$. Final extension was carried out at $72^{\circ} \mathrm{C}$ for 5 minutes.

Seven polymorphic AFLP markers were used in this study too. These 7 primer combinations made 63 polymorphic bands. AFLP reactions were performed as described by Vos et al. (1995) using EcoRI and MseI primers without a selective base in pre-amplification and with three selective bases in amplification. For this purpose, genomic DNA was digested with a pair of restriction endonucleases (EcoR I and Mse I), and the combination of genomic DNA and restriction enzymes were incubated for 12 hours at $30^{\circ} \mathrm{C}$. then double-stranded EcoR I (Eco) and Mse I (Mse) linkers were ligated to the restriction fragments. After that the pre-selective amplification, a subset of all the fragments was amplified, using primers that are complementary to the linker sequences. In the last step, the number of fragments was further reduced by a

Tab. 1. AFLP primers used for QTL mapping

\begin{tabular}{cr}
\hline Primer & Sequence \\
\hline MseI & 5'-GATGAGTCCTGAGTAA-3' \\
\hline EcoRI & 5'-GTAGACTGCGTACCAATTC-3' \\
MseI adaptor & 5'-GACGATGAGTCCTGAG-3' \\
MseI adaptor & 3'-TACTCAGGACTCAT-5' \\
EcoRI adaptor & 5'-CTCGTAGACTGCGTACC-3' \\
EcoRI adaptor & 3'CATCTGACGCATGGTTAA-5' \\
E32-M61 & Eco-AAC+MseCTG \\
E36-M42 & Eco-ACC+MseAGT \\
E39-M39 & Eco-AGA+MseAGA \\
E37-M61 & Eco-ACG+MseCTG \\
E40-M93 & Eco-AGC+MseTTG \\
E35-M90 & Eco-ACA+MseTGT \\
\hline
\end{tabular}

second round of PCR (selective amplification), in which the PCR primers had an additional three selective bases (Meudt and Clark, 2007). The PCR products were separated on denaturing $6 \%$ polyacrylamide gels and the bands were revealed using the silver-staining protocol (Panad et al., 1996). The primers used for AFLP analysis are listed in Tab. 1 .

\section{Linkage map construction and QTL analysis}

A linkage map was constructed using $\mathrm{F}_{2}$ mapping algorithm with QTXb17 Mapmanager (Manly et al., 2001; http://www.mapmanager.org), based on the segregation data of 51 SSR and 63 AFLP loci in the $F_{2}$ population. The related genomic distances (cM) were calculated from recombination values using the Kosambi mapping function (Kosambi, 1944). In order to mapping the QTLs, Composite interval mapping was conducted using QTL cartographer version 2.5 (Basten et al., 1997). To determine empirical significance thresholds for declaring a QTL, 1000 permutations were done to calculate LOD thresholds for each trait at $p=0.05$ and $p=0.01$. Permutation testing (using QTL cartographer) indicated that a logarithm of the odds ratio (LOD) score of 2.5 is suitable threshold for this data.

\section{Results}

\section{Correlation analysis}

According to the results of Duncan's test, a highly significant difference in all traits $(p \leq 0.05)$, between the two parents Domsiah and IR44 were observed (Tab. 2, Fig 1). Tab. 3 also shows the phenotypic (above diagonal) and genotypic variation among the studied traits. All the studied traits had positive correlation with GY. PE had a negative correlation with BPL, FL, FLW, TGW and AL.

Tab. 2. Phenotypic value of parents and $\mathrm{F}_{2}$ population of measured in rice

\begin{tabular}{ccccc}
\hline $\begin{array}{c}\text { Trait } \\
\text { name }\end{array}$ & 'Domsiah' & 'IR44' & $\mathrm{F}_{2}$ & $\mathrm{~T}$ \\
\hline & $\mathrm{m} \pm \mathrm{SD}$ & $\mathrm{m} \pm \mathrm{SD}$ & $\mathrm{m} \pm \mathrm{SD}$ & $\mathrm{m} \pm \mathrm{SD}$ \\
\hline $\mathrm{PH}$ & $110.43 \pm 11.2$ & $128.22 \pm 6.074$ & $132.36 \pm 12.063$ & $-60.7^{* *}$ \\
$\mathrm{BPL}$ & $25.85 \pm 1.87$ & $30.34 \pm 2.64$ & $27.30 \pm 2.95$ & $-16.8^{* *}$ \\
$\mathrm{PE}$ & $5.6 \pm 2.1$ & $4.48 \pm 1.6$ & $5.41 \pm 2.57$ & $-4.30^{* *}$ \\
$\mathrm{NP}$ & $31.71 \pm 1.73$ & $29.3 \pm 3.2$ & $37.39 \pm 2.31$ & $-9.05^{* *}$ \\
\hline $\mathrm{Pd}$ & $35.6 \pm 5.6$ & $40.68 \pm 4.6$ & $37.39 \pm 7.97$ & $-13.36^{* *}$ \\
$\mathrm{FL}$ & $29.7 \pm 2.76$ & $38.61 \pm 3.8$ & $30.43 \pm 4.906$ & $-10.98^{* *}$ \\
\hline $\mathrm{FLW}$ & $1 \pm 0.2$ & $1.1 \pm 0.24$ & $1.18 \pm 0.36$ & $-0.8^{* *}$ \\
\hline $\mathrm{AL}$ & $3.1 \pm 0.87$ & $2.1 \pm 0.36$ & $2.98 \pm 0.68$ & $0.9^{* *}$ \\
\hline $\mathrm{GY}$ & $50.56 \pm 4.1$ & $65.43 \pm 4.3$ & $55.51 \pm 5.098$ & $-24.65^{* *}$ \\
\hline FG & $78.2 \pm 4.3$ & $95.5 \pm 5.6$ & $81.49 \pm 7.59$ & $-45.50^{* *}$ \\
\hline $\begin{array}{c}1000 \text { seed } \\
\text { weight }\end{array}$ & $28.23 \pm 3.2$ & $34.45 \pm 2.2$ & $30.04 \pm 3.1$ & $-14.47^{* *}$ \\
\hline
\end{tabular}

$\mathrm{PH}=$ plant height; $\mathrm{BPL}=$ basal panicle length; $\mathrm{PE}=$ panicle exertion; $\mathrm{NP}=$ number of panicles per plant; $\mathrm{Pd}=$ peduncle length; $\mathrm{FL}=$ flag leaf length; $\mathrm{FLW}=$ flag leaf width; $\mathrm{AL}=$ awn length; $\mathrm{GY}=$ grain yield; $\mathrm{FG}=$ full grain per panicle 
118

Tab. 3. Genotypic (below diagonal) and phenotypic (above diagonal) correlation coefficients among traits in the $160 \mathrm{~F}_{2}$ rice individuals

\begin{tabular}{cccccccccccc}
\hline Trait & PH & BPL & PE & NP & Pd & FL & FLW & AL & GY & FG & TGW \\
\hline PH & 1 & 0.441 & 0.160 & 0.234 & 0.382 & 0.230 & 0.150 & -0.029 & 0.376 & 0.330 & 0.129 \\
BPL & 0.442 & 1 & -0.045 & 0.090 & 0.204 & 0.464 & 0.079 & 0.084 & 0.309 & 0.318 & 0.175 \\
PE & 0.160 & -0.046 & 1 & 0.044 & 0.047 & -0.036 & -0.086 & -0.111 & 0.209 & 0.273 & -0.035 \\
\hline NP & 0.235 & 0.090 & 0.043 & 1 & 0.234 & 0.111 & -0.049 & -0.041 & 0.218 & 0.107 & 0.051 \\
Pd & 0.385 & 0.206 & 0.047 & 0.238 & 1 & 0.142 & 0.089 & -0.031 & 0.124 & 0.074 & 0.004 \\
\hline FL & 0.230 & 0.463 & -0.036 & 0.111 & 0.143 & 1 & 0.199 & 0.097 & 0.253 & 0.160 & 0.088 \\
\hline FLW & 0.179 & 0.103 & -0.101 & -0.051 & 0.102 & 0.240 & 1 & 0.047 & 0.029 & -0.099 & -0.069 \\
AL & -0.030 & 0.085 & -0.113 & -0.041 & -0.031 & 0.098 & 0.056 & 1 & 0.010 & -0.008 & -0.093 \\
GY & 0.383 & 0.314 & 0.208 & 0.223 & 0.127 & 0.257 & 0.034 & 0.005 & 1 & 0.720 & 0.152 \\
\hline FG & 0.331 & 0.320 & 0.275 & 0.109 & 0.074 & 0.160 & -0.119 & -0.008 & 0.734 & 1 & 0.217 \\
TGW & 0.130 & 0.179 & -0.034 & 0.050 & 0.002 & 0.090 & -0.076 & -0.093 & 0.160 & 0.220 & 1 \\
\hline
\end{tabular}

$\mathrm{PH}=$ plant height; $\mathrm{BPL}=$ basal panicle length; $\mathrm{PE}=$ panicle exertion; $\mathrm{NP}=$ number of panicles per plant; $\mathrm{Pd}=$ =peduncle length; $\mathrm{FL}=$ flag leaf length; $\mathrm{FLW}=$ flag leaf width; $\mathrm{AL}=$ awn length; $\mathrm{GY}=$ grain yield; $\mathrm{FG}=$ full grain per panicle; $\mathrm{TGW}=1000$-grain weight

Tab. 4. Putative QTLs for agronomic traits in $\mathrm{F}_{2}$ population derived from the cross of two rice varieties ('Domsiah' $\times$ 'IR44')

\begin{tabular}{|c|c|c|c|c|c|c|c|c|}
\hline Trait & $\mathrm{QTL}^{\mathrm{a}}$ & Flanking markers & Chro & $\mathrm{LOD}^{\mathrm{b}}$ & $a^{c}$ & $\mathrm{~d}^{\mathrm{d}}$ & $\mathrm{d} /|\mathrm{a}|^{\mathrm{e}}$ & $\mathrm{PEV}^{\mathrm{f}}$ \\
\hline \multirow{2}{*}{$\mathrm{PH}$} & qPH-3 & E35-M90-11-E37-M61-11 & 3 & 5.71 & 5.29 & -3.52 & -0.67 & 7.62 \\
\hline & $\mathrm{qPH}-12$ & E40-M93-2-E39-M39-9 & 12 & 3.41 & 3.53 & -4.49 & -1.27 & 2.04 \\
\hline \multirow{2}{*}{ BPL } & qBPL-1 & E40-M93-5-E36-M42-2 & 1 & 2.68 & -1.88 & 0.08 & 0.04 & 14.09 \\
\hline & qBPL-11 & E32-M62-2-E32-M62-7 & 11 & 4.05 & 1.56 & -0.15 & -0.09 & 11.04 \\
\hline $\mathrm{FG}$ & $\mathrm{qFG}-1$ & RM466-RM8097 & 1 & 2.96 & 5.83 & -32.15 & -5.51 & 13.5 \\
\hline PE & qPE-8 & RM8264-RM5720 & 8 & 3.69 & 1.13 & -1.88 & -1.67 & 18.99 \\
\hline \multirow{3}{*}{ TGW } & qGW-3 & E40-M93-6-RM6832 & 3 & 2.64 & 5.44 & -2.79 & -0.51 & 8.4 \\
\hline & qGW-5 & E40-M93-12-E37-M61-6 & 5 & 3.03 & 8.88 & -9.31 & -1.05 & 15.45 \\
\hline & qGW-6 & RM7179-RM5371 & 6 & 5.23 & 11.12 & -1.01 & -0.09 & 28.82 \\
\hline \multirow{2}{*}{ GY } & qGY-2 & RM221-RM236 & 2 & 5.86 & -0.57 & 3.37 & 5.91 & 11 \\
\hline & qGY-11 & E40-M93-9-E36-M42-3 & 11 & 4.36 & -0.70 & -0.76 & -1.09 & 7.1 \\
\hline \multirow{2}{*}{ Pd } & qPd-6 & RM7179-RM5371 & 6 & 3.06 & 3.80 & 0.46 & 0.12 & 12.77 \\
\hline & qPd-8 & E32-M62-5-RM6208 & 8 & 3.01 & -2.55 & 6.10 & 2.39 & 3.78 \\
\hline \multirow{3}{*}{ FL } & qFL-4 & RM261-RM 8212 & 4 & 4.40 & 0.55 & -2.54 & -4.59 & 0.04 \\
\hline & qFL-6 & RM527-E39-M39-4 & 6 & 3.09 & -1.17 & 18.39 & 15.66 & 0.23 \\
\hline & $\mathrm{qFL}-8$ & E35-M90-10-E32-M62-5 & 8 & 2.51 & -4.20 & 4.42 & 1.05 & 11.28 \\
\hline \multirow{2}{*}{ FLW } & qFLW-1 & RM8094-RM466 & 1 & 7.30 & 0.70 & 6.04 & 8.66 & 12.26 \\
\hline & qFLW-2 & RM1358-RM300 & 2 & 5.04 & -0.53 & 3.14 & 5.94 & 10.18 \\
\hline \multirow{2}{*}{$\mathrm{NP}$} & qNP-4 & E39-M39-8-RM1359 & 4 & 2.97 & 1.90 & -1.35 & -0.71 & 13 \\
\hline & qNP-6 & RM7179-RM5371 & 6 & 3.85 & 1.88 & -0.98 & -0.52 & 64 \\
\hline \multirow{2}{*}{$\mathrm{AL}$} & qAL-3 & E40-M93-6-RM6832 & 3 & 3.76 & -1.24 & 1.38 & 1.12 & 19.36 \\
\hline & qAL-5 & E37-M61-6-RM3148 & 5 & 2.99 & -0.70 & 0.89 & 1.27 & 8.33 \\
\hline
\end{tabular}

${ }^{\mathrm{a}} \mathrm{QTL}$ are named by abbreviations plus chromosomal number; ${ }^{\mathrm{b}} \mathrm{Log} 10$ likelihood; ${ }^{\mathrm{C}}$ Additive effect; ${ }^{\mathrm{d}}$ Dominance effect; ${ }^{\mathrm{e}}$ Degree of dominance; ${ }^{\mathrm{f}}$ Percentage of total phenotypic variance explained by the QTL

\section{QTL mapping}

A total of 23 QTLs were mapped for 11 agronomical traits using CIM. These QTLs were located on chromosomes 1, 2, 3, 4, 5, 6, 8, 11 and 12 (Tab. 4, Fig. 2). TWO QTLs were mapped for plant height. qPH-3 with LOD= 5.71, was identified on chromosome 3 at the position of 89.6 cM (between E35-M90-11 and E37-M61-11) and explained $7.62 \%$ of phenotypic variation. The allels from 'DOM' increased plant height for $5.29 \mathrm{~cm}$. the second QTL was located on chromosome 12 at $56.2 \mathrm{cM}$ (between
E40-M93-2 and E39-M39-9). qPH-12 with LOD= 3.41 only explained $2.04 \%$ of phenotypic variation and had a minor effect on this trait. Like the previous QTL, the alleles from 'DOM' increased the plant height by $3.53 \mathrm{~cm}$. Two QTLs were identified for panicle length. qPL-1 was located on chromosome 1 at $156.7 \mathrm{cM}$ (between E40M93-5 and E36-M42-2) and with $\mathrm{LOD}=2.68$ explained $14.09 \%$ of phenotypic variation. The allele from ' $\mathrm{DOM}$ ' increased the PL negatively. The second QTL (qPL-11) with $\mathrm{LOD}=4.05$ was located on chromosome 11 at 62.4 

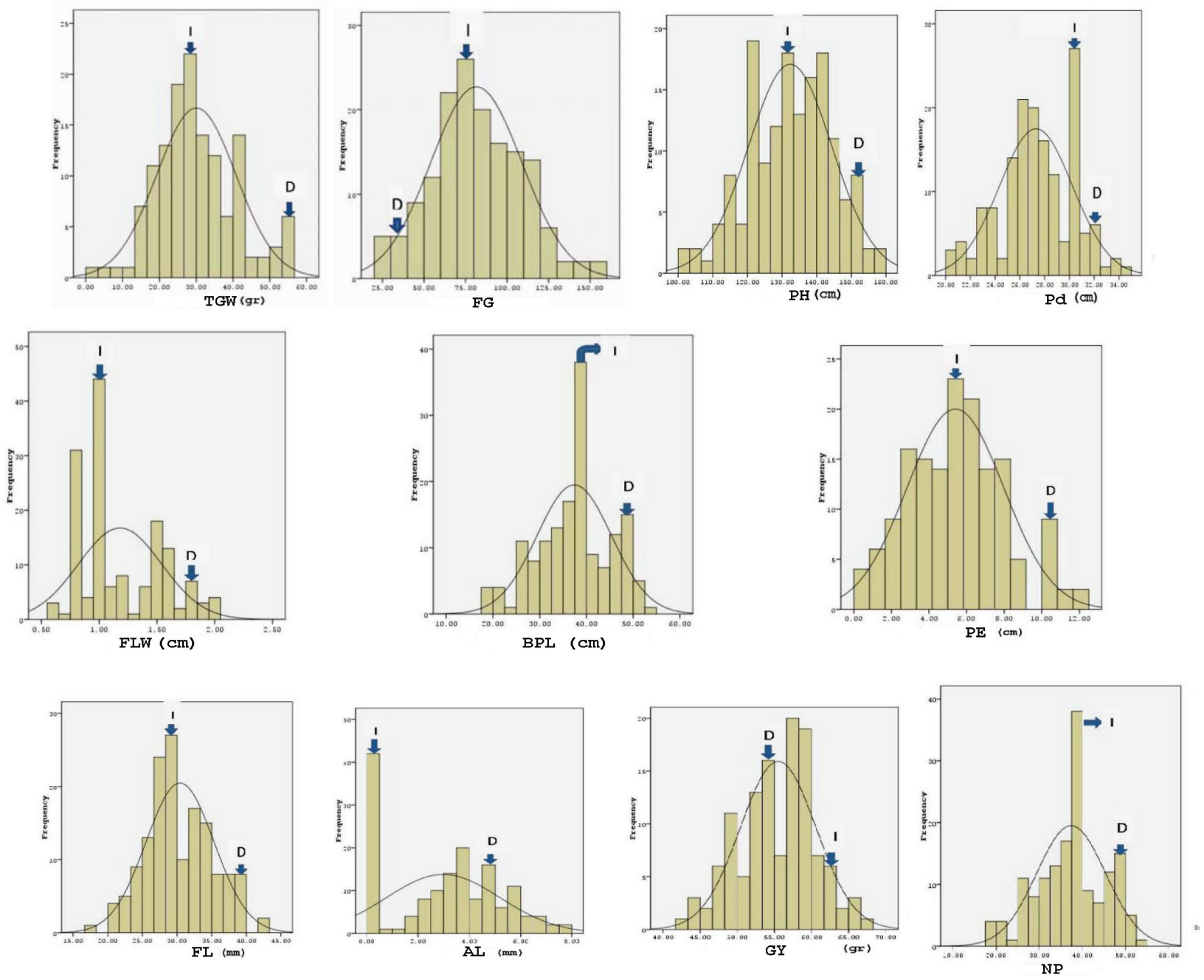

Fig. 1. Frequency distribution of the means of the $160 \mathrm{~F}_{2}$ families for each trait. The parental means of 'IR 44 ' and 'DOM' are indicated by arrows

cM (between E32-M62-2 and E32-M62-7) and explained $11.04 \%$ of phenotypic variation. The 'DOM' alleles increased the PL for 1.56 for this QTL. Both QTLs for this trait were main effect.

QTL of qPE-8 associated with panicle exertion was detected at chromosome 8 at the position of $37.3 \mathrm{cM}$ (between RM8264 and RM5720) with LOD= 3.69. It contributed to $18.99 \%$ of variation and was contributed by 'DOM' with additive effect of $3.69 \mathrm{~cm}$. Two QTLs were associated with number of panicles per plant (NP). The first QTL with $\mathrm{LOD}=2.96$ was located on chromosome 4 at $215.9 \mathrm{cM}$ (between E39-M39-8 and RM1359) and explained $9.58 \%$ of phenotypic variation for this trait. The second QTL with $\mathrm{LOD}=3.85$ was located on chromosome 6 at $1.5 \mathrm{cM}$ (between RM7179 and RM5371). For both QTLs, the alleles from 'DOM' increased the NP by 1.9 and 1.88 respectively. Two QTL were detected to be associated with peduncle length, qPd-6 and qPd-8. The qPd-6 located at $17.5 \mathrm{cM}$ of chromosome 6 closely linked to RM7179 contributing $12.77 \%$ of variation. The donor for the favorable allele was 'DOM' with the additive effect of $3.80 \mathrm{~cm}$. The qPd-8 located at $159.1 \mathrm{cM}$ of chromosome 8 between E32-M62-5 and RM6208. It was contributed by 'IR44' with the additive effect of $2.55 \mathrm{~cm}$.

Two QTLs for flag leaf width were identified on chromosome 1 and 2. The QTLs qFW-1 and qFW-2 with a LOD scores of 7.30 and 5.04 showed the large effects on the FW and explaining 12.26 and $10.18 \%$ of the total phenotypic variance, respectively. In second QTL alleles from 'IR 44 ' decreased FW by $0.53 \mathrm{~mm}$, whereas, for the first QTL that was detected on chromosome 1, allele from 'DOM' increased FW by $0.70 \mathrm{~mm}$. from SPD.

Three QTLs for FL were mapped on chromosomes 4, 6 and 8 respectively. qFL- 4 with a LOD score 4.40 on chromosome 4 at $1.5 \mathrm{cM}$ (between RM261 and RM 8212), showed the low effect on the FL. The second QTL (qFL-6) on chromosome 6 at $45 \mathrm{cM}$ (tightly linked with RM527) also showed the low effect on the FL. Whereas qFL-8 on 
Chr 1

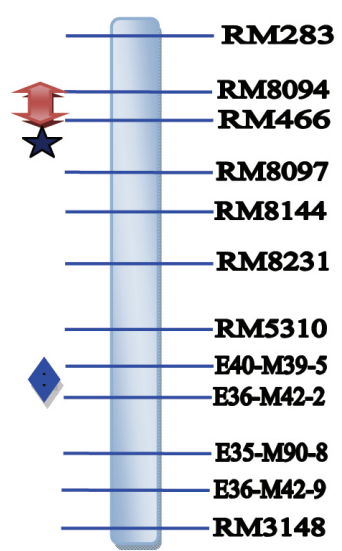

\section{Chr 6}

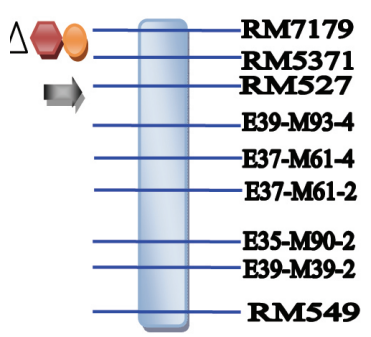

Chr 2

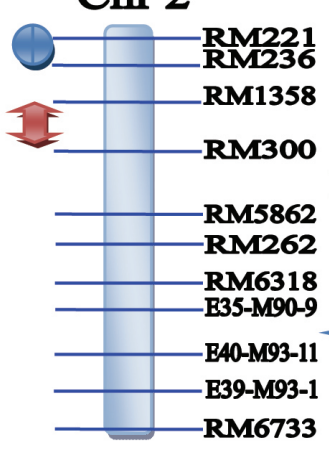

Chr 7

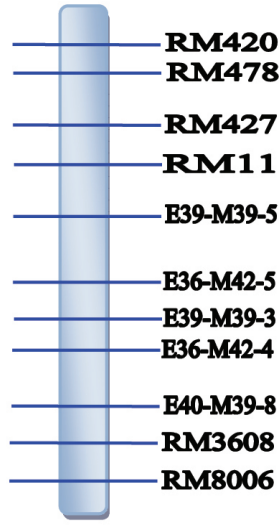

Chr 4

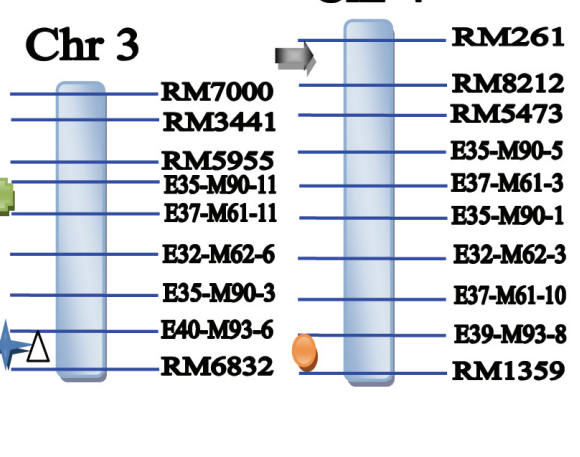

\section{Chr 5}

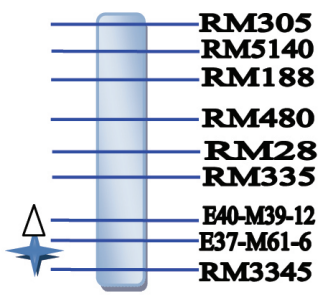

\footnotetext{
$\mathrm{PH}$

$\mathrm{BPL}$

$>P E$

NP

$\mathrm{Pd}$

$\Rightarrow \mathrm{FL}$

1 FLW

$\stackrel{t}{A} \mathrm{AL}$

O $\mathrm{GY}$

穴 FG

$\triangle \mathrm{TGW}$
}

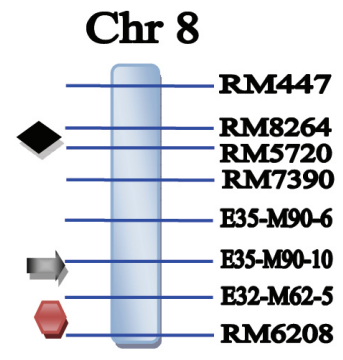

Chr 9

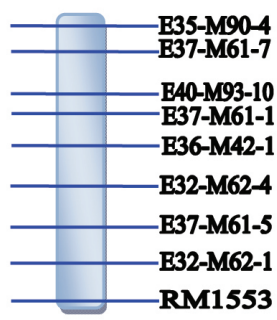

Chr 10

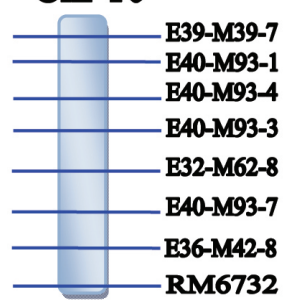

\section{Chr 11}

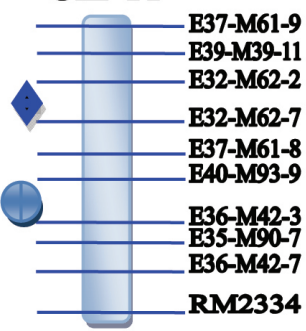

\section{Chr 12}

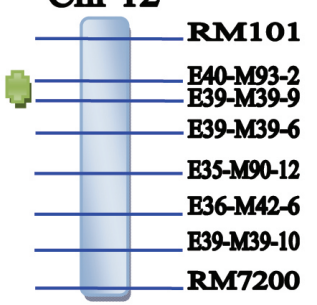

Fig. 2. QTLs that were mapped for: full grain per panicle (FG), flag leaf width (FLW), 1000-grain weight (TGW), number of panicles per plant (NP), flag leaf length (FL), peduncle length (Pd), plant height (PH), panicle exertion (PE), grain yield (GY), basal panicle length $(\mathrm{BPL})$ and awn length $(\mathrm{AL})$ 
chromosome 8 at $144.3 \mathrm{cM}$ (between E35-M90-10 and E32-M62-5) explained $11.28 \%$ of the total phenotypic variance. Two QTLs alleles out of all detected QTLs were from 'IR44' and decreased FL. Two QTLs were mapped for AL. the first QTL on chromosome 3 at $207.2 \mathrm{cM}$ (between E40-M93-6 and RM6832) had major effect on this QTL and explained $19.36 \%$ of phenotypic variance. The second QTL was mapped on chromosome 5 at $210.1 \mathrm{cM}$ (between E37-M61-6 and RM3148). qAL-5 with LOD score of 2.99 , explained $8.33 \%$ of phenotypic variance. For both QTLs, alleles from 'IR 44' decreased AL by 1.24 and $0.7 \mathrm{~mm}$ respectively.

For grain yield, two QTLs were mapped. qGY-2 with $\mathrm{LOD}=5.86$ on chromosome 2 at $1.5 \mathrm{cM}$ (between RM221 and RM236) explained $19.36 \%$ of phenotypic variance for this trait. The alleles from 'DOM' decreased the grain yield by $0.57 \mathrm{gr}$. The second QTL, qGY-11 at $145.9 \mathrm{cM}$ (between E40-M93-9 and E36-M42-3) on chromosome 12 explained $8.33 \%$ of phenotypic variance and had decreasing allele from 'DOM' for grain yield. One QTL was identified for FG. The related QTL was on chromosome 1 at $59.7 \mathrm{cM}$ (between RM466 and RM8097). The alleles from 'IR 44 ' increased the healthy grains by 5.83 . Finally three QTLs were identified for 1000 grain weight. These QTLs were mapped on chromosomes 3, 5 and 6 respectively.

qGW-3 was the minor effect QTL for this trait. This QTL with $\mathrm{LOD}=2.64$ on chromosome 3 at $194.7 \mathrm{cM}$ (between E40-M93-6 and RM6832) only explained 8.5\% of phenotypic variance. 'DOM' alleles increased GW for this QTL. The second QTL (qGW-5) on chromosome 5 at $168.8 \mathrm{cM}$ (between E40-M93-12 and E37-M61-6) with $\mathrm{LOD}=3.03$, explained $15.45 \%$ of phenotypic variance. The last QTL was mapped on chromosome 6 . This QTL (qGW-6) at $8.5 \mathrm{cM}$ (between RM7179 and RM5371) explained $28.82 \%$ of phenotypic variance and was introduced as a major QTL for this trait. For the two last QTLs, alleles from 'DOM' increased the 1000 grain weight by 8.88 and $11.12 \mathrm{gr}$ respectively. Currently, there are still large knowledge gaps on the molecular controls of the basic biological processes related to yield traits. This is especially the case for grain development. With the global development of functional genomic resources, many tools and genetic stocks have now become available for identifying genes underlying these processes (Xing and Zhong, 2010).

\section{Discussion}

Since the early 1990s, numerous studies have identified molecular markers linked to QTLs involved in the inheritance of agronomically important traits in a wide range of crop species. Following the discovery of promising QTLs and identification of molecular markers, MAS has been used to transfer single genes or QTL in various species. However, published results in QTL introgressions through MAS are variable, ranging from successful experiments to those with limited success and even a failure (Semagn, 2010). Yield is a complex trait governed by many loci, it is significant that some loci exert a much greater influence on yield. Single genes are known to enhance yield significantly (about 15 to $20 \%$ on per plant basis) as also shown in studies using transgenics. It is such genes or major effect QTLs that can be targeted for yield improvement. A combination of more than one major yield QTLs can be pooled together in a genotype for increasing yield (Swamy and Sarla, 2008).

In this study, 22 QTLs were mapped for 11 agronomical traits. These QTLs were distributed on chromosomes $1,2,3,4,5,6,8,11$ and 12. Chromosome 6 had the most QTLs (4 QTLs), and after that, chromosomes 1, 3 and 8 were in the second place (3 QTLs on each chromosomes). 2 QTLs were mapped for PH. The first QTL had partial Dominance effect and the second one had over dominance effect. Sabouri and Biabani (2009) mapped 2 QTLs for PH using an $\mathrm{F}_{2.3}$ population derived from a cross between two indica type varieties, Tarommahalli and Khazar. The identified QTLs were mapped on chromosome 3 (2 QTLs). Their study showed that these QTLs have over dominance effect. Zhang et al. (2004) mapped QTLs for plant height on the same chromosomes. Two QTLs were mapped for panicle length. Both of them had partial dominance effect and had high effects on the related trait. Xiao et al. (1998) mapped QTLs for PL using $\mathrm{BC}_{2}$ testcross population (V20A/O. rufpogon//V20B///V20B////Ce64) consisting of 300 families. They have found seven QTLs for this trait, which two of them were on chromosome 1 . Those QTLs explained $14 \%$ of phenotypic variation (same as present study). Sabouri et al. (2010) mapped 4 QTLs for panicle length using $236 \mathrm{~F}_{2.3}$ families derived from the cross of two rice varieties (Gharib $\times$ Sepidroud). They found 1 QTL on chromosome 1 that explained $15.36 \%$ of phenotypic variation. Their QTL had over dominance effect.

One QTL was mapped for PE on chromosome 8. This major effect QTL explained $18.99 \%$ of phenotypic variance. Yue et al. (2008) mapped QTLs related to PE on the same chromosome. They found 1 QTL with $\mathrm{LOD}=5.5$ on chromosome 8 that explained $15.89 \%$ of phenotypic variation. Three QTLs were mapped on chromosomes 3, 5 and 8 respectively. qGW-6 was major effect. Jing et al. (2010) mapped 10 agronomical traits using an advanced backcross $\mathrm{BC}_{3}$ population from a cross between recurrent parent named Yuexiangzhan and donor parent named G52-9. They mapped ten QTLs for the trait of 1000GWt on chromosomes 3, 4, 5, 6 and 11, explaining the phenotypic variance from $3.77 \%$ to $28.67 \%$. Hittalmani et al. (2003) mapped QTLs for TGW on the same chromosomes. Two QTLs were mapped for GY. Both of them had partial dominance effect. They explained the phenotypic variation in range of 8-12\%. Fu et al. (2010) mapped QTLs controlling GY in two generations using 187 SSR 
122

markers. They mapped 4 QTLs for GY on chromosomes $1,2,8$ and 12 . The QTL on chromosome 2 (qGY-2) was minor effect. Two QTLs were identified on chromosomes 3 and 5. These QTLs had over dominance effect. Rahman et al. (2007) mapped QTLs for AL using an $\mathrm{F}_{2: 3}$ population from the cross between 'IR71033' and 'Junambyeo' (a Korean japonica cultivar) consisting of 146 lines. They found three QTLs on chromosomes 4, 5 and 9. qAL-5 with $\mathrm{LOD}=4.09$ explained $12 \%$ of phenotypic variation.

Two QTLs were detected for NP on chromosomes 4 and 6. Both of these QTLs had partial dominance effect. Mocanda et al. (2001) mapped NP related QTLs in a $\mathrm{BC} 2 \mathrm{~F} 2$ population derived from an inter specific cross between Caiapo, an upland Oryza sativa subsp. japonica rice variety from Brazil, and an accession of Oryza rufipogon from Malaysia. They found two QTLs for NP on chromosomes 6 and 11 respectively. For FL, three QTLs were detected on chromosomes 4,6 and 8. All three QTLs had over dominance effect. Two of three QTLs were minor effect. The chromosomal regions of these QTL is as same as Rahman et al. (2007). For FLW two QTLs were mapped. $\mathrm{qFW}-1$ and $\mathrm{qFW}-2$ had over dominance effect and both of them had major effects on this trait.

In general, the number of QTL detected in this study was relatively lower compared to those of other studies. Susanto et al. (2008) believe that It might be due to the size of the population. The larger the mapping population, the more likely to allow detection of more QTL with smaller effect. In most of the cases degree of dominance $(\mathrm{d} /|\mathrm{a}|)$ were high and there was some cases of over dominance toward increased or decreased a trait of interest which can be associated with observed heterosis in $\mathrm{F}_{1}$ hybrids (data not shown). Although, additive and dominance genetic effects could be result from the present cross but, one should take into consideration that epistatic effects and linkage may upwardly bias the dominance, and even partial dominance estimation to become pseudo-over dominance (Sabouri et al., 2010).

\section{References}

Basten CJ, Weir BS, Zeng ZB (2001). QTL cartographer: a reference manual and toturial for QTL mapping. North Carolina State University, 163 p.

BeiBei M, WenJuan C, ZhiHongZ, ZhongLi H, Ping L, LiHuang Z, YingGuo Z (2003). Characterization of QTLs for harvest index and source-sink characters in a $\mathrm{DH}$ population of rice (Oryza sativa L.). Acta Genet Sinica 30(12):1118-1126.

Dellaporta SC, Wood F, Hicks JB (1983). A plant DNA minipreparation: Version II. Plant Mol Biol Repor 1(4):1921.

Fu Q, Zhang P, Tan L, Zhu Z, Ma D, Fu Y, Zhan X, Cai H, Sun C (2010). Analysis of QTLs for yield-related traits in Yuanjiang common wild rice (Oryza rufipogon Griff.). J Genet Genomics 37:147-157.
Grisel JE (2000). Quantitative trait locus analysis. Alcohol Res Health 24(3):169-174.

Gomez SM, Kumar SS, Jeyaprakash P, Suresh R, Biji KR, Boopathi NM, Price AH, Babu RC (2006). Mapping QTLs linked to physio-morphological and plant production traits under drought stress in rice (Oryza sativa L.) in the target environment. Am J Biochem Biotechnol 2:161-169.

Hittalmani S, Huang N, Courtois B, Venuprasad R, Shashidhar HE, Zhuang JY, Zheng KL, Liu GF, Wang GC, Sidhu JS, Srivantaneeyakul S, Singh VP, Bagali PG, Prasanna HC, McLaren G, Khush GS (2003). Identification of QTL for growth and grain yield related traits in rice across nine locations of Asia. Theor Appl Genet 107:679-690.

Jing Z, Qu Y, Chen Y, Pan D, Fan Z, Chen J, Li C (2010). QTL analysis of yield-related traits using an advanced backcross population derived from common wild rice (Oryza rufipogon L). Molec Plant Breed 1(1):1-10.

Kosambi DD (1944). The estimation of map distances from recombination values. Ann Eugen 12:172-175.

Manly KF, Olson JM (1999). Overview of QTL mapping software and introduction to map manager QTL. Mamm Genome 10:327-334.

McCouch SR, Teytelman L, Xu Y, Lobos K, Clare K, Walton M, Fu B, Maghirang R, Li Z, Xing Y, Zhang Q, Kono I, Yano M, Fjellstrom R, De-Clerck G, Schneider D, Cartinhour S, Ware D, Stein L (2002). Development of 2240 new SSR markers for rice (Oryza sativa L.). DNA Res 9:199-207.

Meudt HM, Clarke AC (2007). Almost forgotten or latest practice? AFLP applications, analyses and advances. Trends Plant Sci 12:106-117.

Moncada P, Martínez CP, Borrero J, Chatel M, Gauch HJr, Guimaraes E, Tohme J, McCouch SR (2001). Quantitative trait loci for yield and yield components in an Oryza sativa $\times$ Oryza rufipogon $\mathrm{BC}_{2} \mathrm{~F}_{2}$ population evaluated in an upland environment. Theor Appl Genet 102:41-52.

Panaud O, Chen X, McCouch SR (1996). Development of microsatellite markers and characterization of Simple Sequence Length Polymorphism (SSLP) in rice (Oryza sativa L.). Mol Gen Genet 252:597-607.

Rahman ML, Chu SH, Choi MS, Qiao YL, Jiang W, Piao R, Khanam S, Cho YI, Jeung JU, Jena KK, Koh HJ (2007). Identification of QTLs for some agronomic traits in rice using an introgression line from Oryza minuta. Mol Cells 24(1):16-26.

Sabouri H, Biabani A (2009). Toward the mapping of agronomic characters on a rice genetic map: quantitative trait loci analysis under saline condition. Biotechnology 8(I)144149.

Sabouri A, Toorchi M, Rabiei B, Aharizad S, Momoeni A, Singh RK (2010). Identification and mapping of QTLs for agronomic traits in indica-indica cross of rice (Oryza sativa L.). Cereal Res Communic 38(3):317-326.

Semagn K, Bjørnstad Å, Xu Y (2010). The genetic dissection of 
quantitative traits in crops. Electronic J Biotechnol http:// www.ejbiotechnology.info.

Susanto U, Aswidinnoor H, Koswara J, Setiawan A, Lopena V, Torizo L, Parminder DVS (2008). QTL mapping of yield, yield components, and morphological traits in rice (Oryza sativa L.) using SSR markers. Bul Agron 36(3):188-195.

Swamy BPM, Sarla N (2008). Yield-enhancing quantitative trait loci (QTLs) from wild species. Biotechnol Adv 26:106 120.

Vos P, Hogers R, Bleeker M, Van de Lee T, Hornes M, Frijters A, Pot J, Peleman H, Kuiper M, Zabeau M (1995). AFLP: a new technique for DNA fingerprinting. Nucl Acids Res 23:4407-4414.

Wang J, Wan X, Li H, Pfeiffer WH, Crouch J, Wan J (2007). Application of identified QTL-marker associations in rice quality improvement through a design-breeding approach. Theor Appl Genet 115:87-100.

Wang L, Wang A, Huang X, Zhao Q, Dong G, Qian Q, Sang T, Han B (2010). Mapping 49 quantitative trait loci at high resolution through sequencing-based genotyping of rice recombinant inbred lines. Theor Appl Genet 122(2):327340 .
Xiao J, Li J, Grandillo S, Ahn SN, Yuan L, Tanksley SD, McCouch SR (1998). Identification of trait-improving quantitative trait loci alleles from a wild rice relative, Oryza rufipogon. Genetics 150:899-909.

Xing Y, Zhang Q (2010). Genetic and molecular bases of rice yield. Annu Rev Plant Biol 61:421-442.

Xing YZ, Tang WJ, Xue WY, Xu CG, Zhang Q (2008). Fine mapping of a major quantitative trait loci, qSSP7, controlling the number of spikelets per panicle as a single Mendelian factor in rice. Theor Appl Genet 116:789-796.

Yue B, Xue W, Luo L, Xing Y (2008). Identification of quantitative trait loci for four morphologic traits under water stress in rice (Oryza sativa L.). J Genet Genomics 35:569-575.

Zhang ZH, Li P, Wang LX, Hua ZL, Zhuc LH, Zhua YG (2004). Genetic dissection of the relationships of biomass production and partitioning with yield and yield related traits in rice. Plant Sci 167:1-8. 